\title{
Estudo Inicial do Inventário de Auto-Estima (SEI) - Forma A
}

\author{
Mônica Gobitta ${ }^{123}$ \\ Raquel Soura Lobo Gurzo \\ Pontificica Universidade Católica de Campinas
}

\begin{abstract}
Resumo
No presente estudo buscou-se investigar os índices de precisão do Inventário de Auto-Estima (SEI)-Forma A ,descrever a autoestima do grupo estudado considerando-se as variáveis: gênero e faixa etária nas dimensões estudadas pelo Inventário. Foram participantes deste estudo 142 crianças e adolescentes de dez a 18 anos. Os resultados referentes aos índices de precisão do Inventário mostraram índices satisfatórios quando considerados os resultados da pontuação geral, o mesmo não ocorreu quando a correlação item-total foi submetida às dimensões estudadas. Com relação aos resultados descritivos da auto-estima do grupo estudado, estes apresentaram pequenas diferenças entre as variáveis pesquisadas. Estes resultados levaram a discussões a respeito da adaptação transcultural de instrumentos de avaliação e as conclusões deste estudo apontam para a necessidade de que o instrumento escolhido seja aperfeiçoado em investigações futuras no sentido de melhorar a consistência interna dos itens. Palauras-chave: Auto-estima; avaliação psicológica; adaptação de testes.
\end{abstract}

Initial Study of the Self-Esteem Inventory (SEI) - Form A

Abstract

In this study the aims were to investigate the rates of reliability of the Self-Esteem Inventory (SEI) - Form A and to describe the self-esteem considering the variables gender and age, in the four dimensions studied by the Inventory. The subjects of this study were 142 children and adolescents from ten to 18 years old from a public school. The results referring to the reliability rates of the Self-Esteem Inventory (SEI) - Form A showed that the reliability had pointed satisfactory rates, although the internal consistency analyzed through the item-total correlation indicated problems in same items. This results conduct to discussions concerning the transcultural adaptation of assessment instruments and this study's conclusions point to the need of improving the chosen instruments in future investigations, towards improving the internal consistency of the items. Keywords: Self-esteem; psychological assessment; test adaptation

Abordar cientificamente o tema auto-estima pode não parecer justificável, pois gera a sensação de que, de tão popularizada por livros de auto-ajuda, pelo senso comum e por ter se tornado uma palavra fácil na psicologização das relações humanas, não faz sentido tal empreitada. Porém, como sugere Mruck (1998), pode-se relacionar pelo menos cinco razões para justificar a necessidade de um enfoque científico para estudo da auto-estima: 1) é um construto muito mais complexo do que pode parecer, pois está fortemente associado com outros aspectos da personalidade; 2) está relacionada à saúde mental ou bem estar psicológico; 3) a sua carência se relaciona com certos fenômenos mentais negativos como depressão e suicídio. Para Coopersmith (1967), as pessoas que solicitam ajuda psicológica expressam com freqüência sentimentos de inadequação, pouco valor e ansiedade associada à baixa

${ }^{1}$ Endereço para correspondência: R. Luis Picolotto, 160. Bairro das Palmeiras, Campinas/SP, 13094-570. Fone: (19) 3295 8371. E-mail: gobitta@siomanet.com.

$2 \mathrm{O}$ presente estudo é parte da dissertação de mestrado em Psicologia Escolar (PUC Campinas) da primeira autora, realizada sob orientacão da segunda autora. Agradecemos a colaboração de Patrícia do Carmo Pereira, Carolina F.C. Carvalho, Nilza Catini, Ana Carolina G. Gayotto, Cristina C.M. Pinho, Cristiane M. M. Valle, Maira P.C. Silva, Raquel Tizzee e Tatiana Gazzell integrantes do Laboratório de Avaliação e Medidas Psicológicas LAMP da PUC Campinas, durante a coleta de dados. Agradecemos ainda o apoio fnanceiro do CAPES durante a realização deste trabalho.

Cópias do Inventário podem ser obtidas com a primeira auto auto-estima; 4) é um conceito relevante às ciências sociais. Wells e Marwell (1976) constatam que "a auto-estima está surgindo como um dos indicadores sociais chave na atual análise de crescimento e progresso social” (p.250); 5) elevada relevância social obtida atualmente. Como opina Mruck (1998), "pesquisadores, especialmente aqueles com uma perspectiva sociológica, centram-se agora na compreensão do papel que desempenha a auto-estima no desenvolvimento social" (p.15). Problemas sociais contemporâneos como abuso de drogas, gravidez precoce, fracasso escolar e delinqüência estão freqüentemente associados a este construto.

O tema aparece sendo regularmente criticado por Wyllie (1974), principalmente no que diz respeito à forma como se avalia a auto-estima, mais especificamente os instrumentos utilizados para avaliá-la. Gradualmente, entretanto, foram surgindo pesquisas na área e nos anos noventa uma geração nova de psicólogos sociais esteve atenta para aspectos específicos do auto-conceito (entre eles a auto-estima) e o para o refino de suas medidas (Mruck, 1998).

Entre o final do século IX e início do século XX o conceito de auto-estima parece não ter sido discutido a fundo. Mas, mas se forem consideradas as idéias relacionadas ao conceito de self nos escritos de William James, Charles.H.Cooley, 
Alfred Adler e George Herber Mead, pode-se encontrar as reminiscências deste construto da personalidade.

Os autores aqui citados contribuíram com as suas idéias para a definição do construto auto-estima, tais como forma como o indivíduo elege as suas metas, na visão de W. James, a aceitação de si mesmo em A. Adler, a importância do outro significante enfatizado por C. H. Cooley e G. H. Mead, e a autenticidade do eu em K. Rogers, que constituíram um pano de fundo ao que mais recentemente foi incorporado a este tema (Bednar \& Peterson, 1995). Cada um destes autores contribuiu para a compreensão do conceito de si mesmo e para a definição da auto-estima como o aspecto valorativo e afetivo deste conceito.

A investigação de Rosenberg (1983) é bastante representativa na explicação das condições associadas com a melhora e com a diminuição da auto-estima. Coopersmith (1967), realizou amplo estudo sobre auto-estima intitulado "Os Antecedentes da Auto-Estima". Este autor tem sido tradicionalmente citado em todos os trabalhos de revisão do construto e na maioria dos estudos empíricos relacionados ao tema (Andrew \& Tracy, 1996; Baumrind, 1967; Bednar \& Peterson, 1995; Bracken, 1996; Buss, 1995; Coll, Palacios \& Marchesi, 1995; Friedman, Gettys \& Rogers, 1975; Harter, 1993; Martins, 1997; Mruck, 1998; Nisbet, 1996; Powers, Singer \& Sowers, 1996; Sanches, Jiménez \& Merino, 1997; Souza, 1978; Wyllie, 1974).

Coopersmith (1967) estudou as condições e experiências concretas que fortalecem ou debilitam a auto-estima, empregando tradicionais métodos psicológicos, particularmente mediante a observação controlada Considerou que as maiores relevâncias para o seu estudo são as indicações de que dominação de crianças, rejeição e punição severa resultam em auto-estima rebaixada. Sob tal condição, as crianças experimentam menos o amor e sucesso, e tendem a ficar geralmente submissas e passivas (embora mudando de comportamento, ocasionalmente, para o oposto extremo de agressão e dominação). Crianças criadas sob tais circunstancias, segundo este autor, têm menor probabilidade de serem realistas e efetivas no seu dia-a-dia, e têm mais probabilidade de manifestar padrões de comportamento anticonvencionais.

Um exemplo de como Coopersmith (1989) encara tais influências é a seguinte declaração: “... crianças não nascem preocupadas em serem boas ou más, espertas ou estúpidas, amáveis ou não. Elas desenvolvem estas idéias. Elas formam auto-imagens... baseadas fortemente na forma como são tratadas por pessoas significantes, os pais, professores e amigos" (p. 2).

Coopersmith (1967) atentou para o fato de que pessoas que procuram ajuda psicológica, freqüentemente, "se vêem como impotentes e inferiores ou ainda incapazes de melhorar a situação, além de lhes faltar os recursos internos para tolerar ou reduzir a ansiedade prontamente despertada por eventos cotidianos e tensão" (p. 3). Lembra o autor que estudos clínicos demonstram, repetidamente, que fracassos e outras condições que ameaçam expor insuficiências pessoais são, provavelmente, a causa principal de ansiedade. Segundo ele, "ansiedade e auto-estima estão proximamente relacionadas: se for a ameaça que libera ansiedade, como aparece teoricamente, é a estima da pessoa que está sendo ameaçada" (p. 4).

Coopersmith (1967) refere-se, também, a estudos que indicam que:

"Uma pessoa com auto-estima alta mantém uma imagem bastante constante das suas capacidades e da sua distinção como pessoa, e que pessoas criativas têm alto grau de auto-estima. Estas pessoas com auto-estima alta também têm maior probabilidade para assumir papéis ativos em grupos sociais e efetivamente expressam as suas visões. Menos preocupados por medos e ambivalências, aparentemente se orientam mais diretivamente e realisticamente às suas metas pessoais" (p. 4).

Finalmente, a definição de auto-estima do autor citado é:

“... a avaliação que o indivíduo faz, e que habitualmente mantém, em relação a si mesmo. Expressa uma atitude de aprovação ou desaprovação e indica o grau em que o indivíduo se considera capaz, importante e valioso. Em suma, a autoestima é um juízo de valor que se expressa mediante as atitudes que o indivíduo mantém em face de si mesmo. É uma experiência subjetiva que o indivíduo expõe aos outros por relatos verbais e expressões públicas de comportamentos" (Coopersmith, 1967, pp. 4-5).

Uma outra questão importante para Coopersmith era: "quais são as características dos outros significantes que alimentam positiva ou negativamente a auto-estima?" (Bednar \& Peterson, 1995). Para avaliar este aspecto, Coopersmith (1967) dividiu as auto-avaliações dos sujeitos em quatro áreas de avaliações subjetivas: pais, amigos, escola e si-mesmo ou eu geral. Dentro destas áreas o indivíduo pode variar de acordo com sexo, idade e outras condições de definição de papéis. Alguns fatores que determinam a auto-avaliação foram elencados por Coopersmith (1967): a) o valor que a criança percebe dos outros em direção a si, expresso em afeto, elogios e atenção; b) a experiência da criança com sucessos ou fracassos; c) a definição individual da criança de sucesso e fracasso, as aspirações e exigências que a pessoa coloca a si mesmo para determinar o que constitui sucesso; e, d) a forma da criança reagir a críticas ou comentários negativos.

Coopersmith (1967) realizou estudo correlacional entre variáveis como condição econômica da família, grau de 
instrução, localização geográfica, classe social, ocupação do pai ou mãe, ou presença constante da mãe em casa. Ele concluiu, a respeito disso, que não existem correlações significantes entre estes fatores. O que ele constatou como significativo para a formação do "eu" foi o relacionamento entre a criança e os adultos importantes de sua vida. Ele encontrou cinco condições que contribuem para melhorar a auto-estima da criança: a) experimentar uma total aceitação de seus pensamentos, sentimentos e valores pessoais; b) estar inserida num contexto com limites claramente definidos, desde que sejam justos e não opressores; c) os pais não usarem de autoritarismo e violência para controlar e manipular a criança, bem como não humilhar, nem a ridicularizar; d) os pais devem manter altos padrões e altas expectativas em termos de comportamentos e desempenhos da criança; e, e) os pais devem apresentar um alto nível de auto-estima, pois eles são exemplos vivos do que a criança precisa aprender. Baumrind (1967) estudou atitudes parentais e auto-estima e constatou características similares às que Coopersmith descreve, ou seja, a correlação entre auto-estima alta nas crianças e aceitação e respeito à individualidade por parte dos pais, dentro de uma postura de autoridade e firmeza.

\section{Avaliação e Medidas em Auto-Estima}

Azevedo, Almeida, Pasquali e Veiga (1996) referemse às dificuldades freqüentemente enumeradas a propósito dos testes psicológicos, como maiores ou mais sentidas em países onde faltam instrumentos devidamente validados e padronizados. Segundo estes mesmos autores, apesar dos problemas enfrentados no Brasil, como "falta de recursos para pesquisa, poucos pesquisadores na área, menor investimento das escolas que formam psicólogos, pouca sensibilidade dos organismos de psicólogos para os problemas enunciados ou o fraco intercâmbio com colegas dentro e fora do Brasil com interesses na avaliação psicológica e outros" (p. 215), é observada uma fase de alta produção na área, devido aos laboratórios de desenvolvimento de pesquisa psicométrica que têm enfatizado a realização de estudos centrados na construção e validação de instrumentos.

Em recente trabalho realizado por Almeida, Prieto; Muñiz e Bartran (1998), investigando o uso de testes em Portugal, Espanha e Iberoamérica, os autores chamam a atenção para a importância de associações internacionais de psicólogos da área de avaliação psicológica, como a Internacional Test Commission - ITC (Bartram, 1999). $\mathrm{Na}$ intenção de estabelecer diretrizes para a adaptação transcultural de testes psicológicos e educacionais, bem como para suprir a literatura quase incompleta de técnicas para guiar este processo, Hambleton (1994), sob a chancela Psicologia: Reflexão e Crítica, 2002, 15(1), pp. 143-150 da Internacional Test Commission - ITC, descreve algumas para adaptação de testes ou instrumentos educacionais e psicológicos.

O uso do modelo de Brislin (Guzzo, 1999) de tradução pelo método da "back-translation" é recomendado pelo comitê acima mencionado, e refere-se a um processo que possibilita bons resultados de adaptação, conforme demonstrou Guzzo (1999). O processo consiste em a) tradução do instrumento da versão original para a língua a que se destina a adaptação; b) a volta para a versão original, por meio da primeira tradução; c) ambas versões na língua original devem ser, então, analisadas por um grupo de juizes tradutores; e d) uma análise estatística deve ser feita, para estabelecer a validade da tradução. No caso de alguns itens não serem válidos, uma segunda fase pode ser prevista, repetindo-se o processo para o estudo dos itens fracos.

A auto-estima e o auto-conceito são construtos teóricos muito semelhantes e embora a sua importância para o estudo da Psicologia, notadamente da personalidade, seja reconhecida, a literatura revela confusão conceitual e dificuldades na definição de ambos. Igualmente, os instrumentos comumente utilizados para avaliar a auto-estima estão articulados e, em outros casos, confundem-se com instrumentos que avaliam o auto-conceito.

Relacionadas com os problemas de avaliação da autoestima estão as observações de Mruk (1998), que agrupou estas dificuldades em dois tipos básicos. O primeiro consiste em problemas decorrentes das singularidades da auto-estima como fenômeno; "estes aspectos incluem o problema da definição, o vínculo entre auto-estima e muitos outros aspectos relacionados com o selfe certas características intrínsecas da auto-estima. As outras dificuldades procedem tanto das expectativas psicológicas como sociológicas, a presença de uma grande diversidade de métodos de investigação usados para o estudo do fenômeno e algumas dificuldades em relação à validade”. (p. 37)

Fatores que estão relacionados à construção de um instrumento para medir a auto-estima são indicados por Mruk (1998) como dificuldades na investigação deste construto da personalidade, entre os quais destaca: a) medir o que é correto, ou seja, o instrumento deve medir a auto-estima, e não outra coisa; b) fatores dinâmicos: um bom teste de auto-estima deve ser multifacetado, capaz de captar todas as situações particulares da vida do sujeito que tem alta ou baixa auto-estima; c) problemas de normatização relativos aos auto-informes; d) previsão de recursos que minimizem o fato de que a maioria das pessoas se pontua mais favoravelmente nas qualidades positivas e menos desfavoravelmente nas negativas; e e) os testes devem ter meios para identificar as atitudes 
defensivas, comumente encontradas em auto-informes de auto-estima (Mruk, 1998).

Em recente pesquisa realizada por Guzzo, Gayotto, Messias e Silva (1998) constatou-se 2599 artigos com palavra chave sef-concept (auto-conceito); desses, 309 artigos falam também de self-esteem (auto-estima) no banco de dados do PsycLIT, entre 1991 a 1997. Nos artigos nos quais foram encontradas referências a estudos com auto-estima foram mais citados os seguintes instrumentos de medida: Tenesse Self-Concept Scale (TSCS) (Fitts, 1965), Self-Esteem Inventory Inventário de Auto-Estima (SEI) Forma-A (Coopersmith, 1989), Piers-Harris Children's Self-Concept Scale (PHSCS) (Piers, 1984), Self-Description Questionaire I (SDQ I) (March, 1988); Self-Description Questionaire II (SDQ II) (March, 1990), Rosenberg Self-Esteem Scale (Rosenberg, 1979), Self-Esteem Index (Brow \& Alexander, 1991), entre outros (Guzzo, Gayotto, Messias \& Silva, 1998). No que respeita a revisão da literatura nacional, observam-se poucas referências à adaptação, para a realidade brasileira, de instrumentos que avaliem a auto-estima. Martins (1997) constatou que existem alguns instrumentos de origem norte-americana traduzidos adaptados para a realidade brasileira: "How I see my self", de Ira Gordon (adaptada por Popovic, Esposito \& Cruz, 1973) e Escala de Auto-Estima de Janis e Field (Martins, 1997).

Dentre os instrumentos utilizados para a avaliação da auto-estima está o "SEI-Self-Esteem Inventory (SEI) Forma A", desenvolvido por Coopersmith (1989), que por apresentar boas qualidades psicométricas em estudos anteriores e por ser um dos mais citados na literatura, foi selecionado para que neste estudo seja adaptado à realidade brasileira. Possibilitando que, em estudos posteriores, possa ser realizada a sua validação.

\section{Método}

\section{Participantes}

Foram participantes do presente estudo 142 alunos, de quinta a oitava série, dos quais $64(45,1 \%)$ eram do gênero feminino e $78(54,9 \%)$ do gênero masculino, de uma escola da rede estadual de ensino fundamental, situada no perímetro urbano do município de Campinas (SP). Enquanto nos estudos estrangeiros os autores remetemse diretamente às séries para fazer referência à idade, no Brasil a situação é diferente, pois a distorção idade/série é acentuada, dos alunos estudados, 41 (28,9\%) estão com idade acima da esperada para a série, gerando, então, um grupo de alunos de idades não uniforme por conseqüência de retenções. Na sexta série encontra-se um número maio de sujeitos e, também, uma incidência maior de distorção idade/série é notada, pois 30 sujeitos (52,7\%) estão fora da idade esperada para a série. Da mesma forma, na oitava série, 19 sujeitos (54,5\%) estão com idade um pouco abaixo, ou muito superior para o esperado para a série. $\mathrm{Na}$ quinta série, os sujeitos que apresentam distorção idade/série são $8(28,6 \%)$ e na sétima série apenas quatro $(18,2 \%)$ estão com idade inferior, e nenhum se encontra em idade superior.

Optou-se por aplicar o instrumento em todos os estudantes das séries citadas, com o objetivo de delimitar a idade dos sujeitos a partir dos dez anos, pois nesta idade, segundo Coopersmith (1989) o indivíduo aparenta ter experiências suficientes e habilidade para pensar abstratamente e fazer avaliações gerais de suas potencialidades. Com relação, ainda, à faixa etária a amostra contou com a seguinte divisão: 33 (23,2\%) alunos encontramse na faixa dos dez aos 11 anos e 11 meses, 70 (49,3\%) encontram-se na faixa dos 12 aos 13 anos e 11 meses, 28 $(19,7 \%)$ entre 14 e 15 anos e 11 meses, dez (7,1\%) entre 16 e 17 anos e 11 meses, e apenas $1(0,7 \%)$, está entre os 18 e 19 anos e 11 meses. Pode-se notar que a maioria dos alunos está entre a faixa de dez a 13 anos e 11 meses, com 103 $(72,5 \%)$.

\section{Material}

Para o presente estudo foram utilizados os seguintes instrumentos:

Self-Esteem Inventory (SEI)-Form A, versão para alunos. No presente trabalho será designado como Inventário de Auto-estima (SEI) - Forma A;

Folhas de Respostas do Inventário de Auto-estima (SEI) - Forma A e Escala de Avaliação do Comportamento (BRF).

Os instrumentos citados foram traduzidos da língua inglesa para adaptação transcultural no âmbito do Laboratório de Avaliação e Medidas Psicológicas -LAMP.

Tais instrumentos foram originalmente desenvolvidos por Stanley Coopersmith $(1967,1989)$ que elaborou um Inventário com 50 itens. Foram, ainda, incorporados o que o autor chamou de oito itens de desejabilidade social que avaliam as atitudes defensivas, comumente encontradas em auto-informes de auto-estima. Os 50 itens do instrumento estão divididos em quatro dimensões: eu geral (26 itens), grupo social (oito itens), família (oito itens) e escola (oito itens).

Os 58 itens do SEI-Forma A são avaliados por duas possibilidades de respostas, "tem a ver comigo" ou "não tem a ver comigo". Para os itens negativos que forem respondidos com "não tem a ver comigo", deve-se considerar uma resposta correta. No caso dos itens positivos respondidos com "tem a ver comigo", deve-se considerar uma resposta correta. Os itens falsos são sempre pontuados 
separadamente, ou seja, as respostas a estes itens nunca devem ser incluídas no Total Possível de Pontuação da auto-estima. Para pontuar estes itens, soma-se um ponto para cada item respondido com "tem a ver comigo". As quatro dimensões podem ser pontuadas separadamente.

Em seu estudo Coopersmith (1967) ressalta que a forma final do Inventário foi administrada nas quintas e sextas séries $(N=87)$, a meninos e meninas. A pontuação variou de 40 a 100 , com média de 82,3 e $(d p=11,6)$. A pontuação média para os homens foi $81,3(d p=12,2)$. Para as mulheres foi $83,3(d p=16,7)$. A diferença entre a pontuação média de meninos e meninas não foi significante. A distribuição foi inclinada em direção à alta auto-estima.

A Folha de Resposta do Inventário de Auto-estima (SEI) - Forma A, apresenta campo destinado à coleta de dados para caracterização dos sujeitos, com perguntas sobre: identificação, sexo, data de nascimento, escola, série que freqüenta e nível de escolaridade dos pais.

\section{Procedimento}

As aplicações aos alunos do Inventário de Auto-estima (SEI)-Forma A foram realizadas coletivamente. Em todos os momentos de coleta de dados o caráter sigiloso do procedimento foi claramente mencionado. A diretora da escola prontificou-se em informar aos pais dos alunos sobre a realização da pesquisa, para que estes estivessem cientes.

\section{Resultados}

O índice de precisão do Inventário de Auto-Estima (SEI) Forma A $(N=142)$ obteve valor $\mu=0,73$. Porém, nas dimensões estudadas pelo instrumento os resultados não são satisfatórios ("eu geral" $=65$; "grupo social" $=27$; "família" =38; "escola" =34). Na Tabela 1 pode-se visualizar o resultado da análise da correlação item-total (rit), com o alpha de Cronbach das dimensões e com os mesmos índices no Inventário Geral, ou seja, nos 50 itens estudados pelo Inventário ("eu geral”, "família”, "escola”, "grupo social"), distribuídos em quatro intervalos: itens com rit $<0,20$; itens com $0,20<$ rit $<0,30$; itens com $0,30<$ rit $<0,40$ e itens com rit $>0,40$.

Para a análise dos resultados gerais obtidos pelos sujeitos, relativamente à variável independente gênero, foi considerada a somatória dos pontos totais (SEI Geral). $\mathrm{Na}$ Tabela 2 pode-se observar a classificação da autoestima em função dos quartis. O primeiro quartil referese à pontuação até 60 (auto-estima baixa); o segundo quartil refere-se à pontuação de 61 a 70 (auto-estima média baixa); o terceiro quartil refere-se à pontuação de

Tabela 1. Índice a de cada Dimensão e Freqüência dos Ítens com Índice de Correlação Item-Total Geral do Inventário de Auto-Estima (SEI) - Forma A, Agrupados em Quatro Intervalos

\begin{tabular}{lrrcccc}
\hline Dimensões & ítens & $a$ & \multicolumn{4}{c}{ Freqüência da correlação item-total geral } \\
\hline & & \multicolumn{6}{c}{$<0,20$} & 0,20 a 0,30 & 0,30 a 0,40 & $>0,40$ \\
Eu Geral & 26 & 0,65 & 12 & $F$ & F & $F$ \\
Grupo Social & 8 & 0,27 & 6 & 7 & 4 & 3 \\
Família & 8 & 0,38 & 3 & 1 & 1 & 2 \\
Escola & 8 & 0,34 & 7 & 1 & \\
\end{tabular}

Coeficiente de precisão $a=0,73$

Tabela 2. Pontuação dos Participantes por Sexo

\begin{tabular}{|c|c|c|c|c|c|c|c|c|}
\hline \multirow[t]{2}{*}{ Quartis } & \multicolumn{6}{|c|}{ Sexo } & \multirow{2}{*}{\multicolumn{2}{|c|}{ ftotal $\%$}} \\
\hline & $\sigma$ & & $\%$ & Q & & $\%$ & & \\
\hline Alta (79 a 100) & 17 & 22 & 57 & 13 & 20 & 43 & 30 & 21 \\
\hline Média Alta (71 a 78) & 20 & 26 & 59 & 14 & 22 & 41 & 34 & 24 \\
\hline Média Baixa (61 a 70) & 20 & 26 & 53 & 18 & 28 & 47 & 38 & 27 \\
\hline Baixa (até 60) & 21 & 26 & 52 & 19 & 29 & 47 & 40 & 28 \\
\hline Total por sexo & & 78 & & & 64 & & 142 & 100 \\
\hline
\end{tabular}

Psicologia: Reflexão e Crítica, 2002, 15(1), pp. 143-150 
71 a 78 (auto-estima média alta) e o quarto quartil referese à pontuação de 79 a 100 (auto-estima alta).

Considerando, portanto, todos os sujeitos avaliados pelo SEI $(N=142)$ foi constatado que o quartil com a maior freqüência foi o referente à auto-estima baixa com $40(28 \%)$ sujeitos, sendo $21(52 \%)$ do sexo masculino e $19(48 \%)$ do sexo feminino. Vê-se que na amostra de sujeitos do sexo masculino houve uma discreta predominância de alunos ( $n=21$ ou $27 \%$ ) no quartil auto-estima baixa. Quanto

Tabela 3. Pontuação dos Participantes por Faixa Etária

\begin{tabular}{lccccccccccccc}
\hline \multirow{2}{*}{ Quartis } & \multicolumn{1}{c}{ Idade (anos) } & \multirow{1}{*}{ total } & \multirow{2}{*}{$\%$} \\
\cline { 2 - 11 } & 10 a 12 & $\%$ & 13 a 15 & $\%$ & 16 a 18 & $\%$ & & \\
\hline Alta (79 a 100) & 14 & 20 & 47 & 14 & 22 & 47 & 2 & 18 & 7 & 30 & 21 \\
Média Alta (71 a 78) & 17 & 25 & 50 & 15 & 24 & 44 & 2 & 18 & 6 & 34 & 24 \\
Média Baixa (61 a 70) & 13 & 19 & 26 & 23 & 36 & 52 & 2 & 18 & 5 & 38 & 27 \\
Baixa (até 60) & 24 & 35 & 60 & 11 & 17 & 27 & 5 & 45 & 12 & 40 & 28 \\
Total por idade & & 68 & & & 63 & & & 11 & & 142 & 100
\end{tabular}

Tabela 4. Pontuação Média por Dimensão do (SEI)- Forma A (N=142)

\begin{tabular}{lrrll}
\hline Dimensões & $m$ & $d p$ & Mínimo & Máximo \\
\hline Família & 0,57 & 0,19 & 0,13 & 0,88 \\
Escola & 0,60 & 0,19 & 0,13 & 1 \\
Grupo social & 0,74 & 0,17 & 0,25 & 1 \\
Eu geral & 0,72 & 0,14 & 0,35 & 1 \\
\hline
\end{tabular}

aos sujeitos do sexo feminino, houve discreta predominância no quartil auto-estima baixa com 19 (29\%).

A tabela 3 apresenta a pontuação geral dos sujeitos por faixa etária. Pode-se verificar no quartil auto-estima baixa que dos 40 (28\%) sujeitos agrupados neste quartil, $24(60 \%)$ estavam na faixa etária de dez a 12 anos. Do total de sujeitos com auto-estima média baixa $23(52 \%)$ estavam na faixa de 13 a 15 anos. Entre os que apresentaram auto-estima média alta $25(50 \%)$ eram pertencentes à faixa dos dez a 12 anos No quartil referente à auto-estima alta, houve uma distribuição uniforme entre os sujeitos de dez a 15 anos com 28 (94\%)

Considerados todos os sujeitos avaliados por meio do SEI - Forma A $(N=142)$, conforme dados da tabela 4, a dimensão que obteve pontuação média mais elevada foi "grupo social" (74), seguida por "eu geral" (72), "escola" (60), e por último, a dimensão referente à "família" (57).

No tocante ao gênero, observou-se que a dimensão "grupo social" aparece com maior pontuação [masculino (73) e para feminino (70)]. A dimensão "escola" apresentou (76) e feminino (73)], seguida pela "eu geral" [masculino pontuação mais baixa para os sujeitos do sexo masculino
(58) e mais alta para os do sexo feminino (63). Na dimensão "família", a pontuação foi mais alta para os sujeitos masculinos (59) do que para os sujeitos femininos (56). Com relação ao efeito da diferença de médias observadas entre os sexos, o nível de significância obtido através do teste T foi: $t=0,738, g l=139, p=0,462$.

Quando considerada a variável idade, foi constatado que na faixa etária de 13 a 15 anos os sujeitos apresentaram pontuação mais elevada em todas as dimensões ["grupo social” (78), "eu geral” (74), "escola" (61) e "família" (59)]. Situaram-se abaixo destas as faixas etárias de dez a 12 anos [“grupo social” (72), "eu geral” (70), "escola" (63) e "família" (57)] e de 16 a 18 anos ["grupo social" e "eu geral" (74), "família" (52) e "escola" (48)].

\section{Discussão e Conclusão}

Estudos com este inventário não foram produzidos no Brasil, apesar de tratar-se de um instrumento citado com freqüência na literatura nacional e internacional, tendo sido estudado por diversos pesquisadores, para fins clínicos ou institucionais, bem como para investigações científicas. No Brasil, observa-se que as pesquisas de avaliação da auto-estima são menos específicas no que diz respeito à auto-estima em si, e mais focalizadas ao auto-conceito e à auto-imagem, sendo, geralmente, usados instrumentos inadequados à avaliação da auto-estima. 
Com relação à precisão do Inventário de Auto-Estima (SEI)-Forma A, é importante salientar que tais resultados devem ser analisados sob a perspectiva de um estudo inicial com dito instrumento. Os resultados obtidos apontam índices de precisão considerados razoáveis. Todavia, quando observados os resultados da correlação item-total, foram constatados itens indicando baixa consistência interna. Estas devem ser as principais preocupações nos estudos subseqüentes. Isto é, convém considerar a possibilidade de uma melhor adaptação transcultural do instrumento, e até a supressão de itens que revelem baixa consistência interna, e que não estejam medindo o que o inventário se propõe a medir.

Comparativamente, o resultado relativo à precisão do SEI Geral obtido no presente estudo não é equivalente ao resultado apresentado por Coopersmith (1967), que, em seu estudo inicial, obteve um índice de precisão de 0,88 . Neste estudo o autor utilizou o método de testereteste para uma amostra de 50 crianças (com cinco semanas de intervalo) e em outra amostra de 56 crianças (com três anos de intervalo) obteve o índice de 0,70.

Com respeito à precisão, em função das quatro dimensões estudadas pelo instrumento, é digna de nota a pesquisa de Donaldson (Coopersmith, 1989). Este estudo valeu-se de uma amostra de 643 crianças de escola pública norte-americana de terceira à oitava série, de diferentes níveis sócio-econômicos, e a correlação encontrada entre as dimensões estudadas pelo SEI foi de 0,02 a 0,52.

Outra investigação sobre a consistência interna do Inventário de Auto-Estima (SEI)-Forma A, realizada por Kimball (Coopersmith, 1989), que aplicou o instrumento em 7600 crianças de escolas públicas norte-americanas, incluindo todas as classes sócio-econômicas e grupos étnicos diversos, resultou em índices de precisão entre 0,87 e 0,92.

Com respeito à pontuação (SEI Geral) dos sujeitos, verificou-se maior incidência de sujeitos no quartil AutoEstima Baixa. Quanto ao gênero, os sujeitos do sexo masculino e feminino se distribuíram com maior freqüência nos quartis inferiores. Considerando-se as dimensões os resultados foram sutilmente diferentes, prevalecendo maior na pontuação média do sexo feminino na dimensão "escola", quando comparada ao sexo masculino. Nas demais dimensões o sexo masculino apresentou maior média. Porém, estas diferenças não foram consideradas significativas estatisticamente, ou seja, estas diferenças podem ter surgido ao acaso.

De acordo com as informações disponíveis na literatura, a maioria dos pesquisadores detectou pequenas diferenças entre média e desvios padrão na pontuação do SEI Geral e nas dimensões referentes aos sexos masculino e feminino, inclusive no estudo de Coopersmith (1967), as diferenças Psicologia: Reflexão e Crítica, 2002, 15(1), pp. 143-150 não foram significativas. Todavia, muitos investigadores observaram diferenças entre os gêneros nas respostas do Inventário de Auto-Estima (SEI)-Forma A que foram acima do padrão do inventário (Coopersmith, 1989). Many (Coopersmith, 1989) constatou a falta de significância entre diferenças nas médias de estudantes femininos e masculinos encontradas no seu estudo, com o Inventário de Auto-Estima (SEI)-Forma A. Por outro lado, Conwan, Altmann, e Pysh (1978) constataram que sujeitos do sexo feminino na $4^{a}$ série $(N=175)$ apresentaram maior pontuação nos itens de desejabilidade social e mostraram mais uniformidade na pontuação relativa à "grupo social" na auto e na heteroavaliação da auto-estima. Constataram, também, que meninas na quarta série, com idade entre oito e 11 anos obtiveram maiores pontos em auto-estima e pareceram experimentar mais sucesso acadêmico do que os meninos. Para Mruck (1998), é consenso que "o gênero é capaz de influir em certo grau sobre a auto-estima e que esta influência se produz numa direção razoavelmente preditiva. Em termos de estrutura geral, as mulheres de nossa sociedade parecem inclinar-se para os componentes de merecimento de auto-estima (serem valorizadas em termos de aceitação ou rejeição) e os homens tendem a inclinar-se para a dimensão de competência (êxito e fracasso)". (p. 77)

Ainda considerando-se os resultados do SEI Geral, os estudantes mais novos apresentaram pontuação predominante no quartil auto-estima baixa, sendo que aqueles com idades intermediárias apresentaram-se em maior número no quartil auto-estima média baixa, e os mais velhos se concentraram no quartil auto-estima baixa. Estas diferenças contrariam o que é habitualmente encontrado na literatura, que refere auto-estima mais alta aos grupos mais jovens, e podem ser atribuídas ao acaso ou ao fato do instrumento não ter discriminado com precisão tal variável.

Com relação à precisão do Inventário de Auto-Estima (SEI)-Forma A, os resultados obtidos apontam índices considerados razoáveis. Ao analisar a consistência interna através da correlação item-total, foi constatada a existência de itens pouco consistentes, considerados fracos. Quando considerados os resultados na correlação item-total em cada dimensão (eu geral, escola, grupo social e família), os índices também não foram satisfatórios.

É importante salientar a relevância dos estudos de adaptação transcultural de instrumentos psicométricos, pois os mesmos são, em muitos casos, desenvolvidos em contextos culturais, econômicos e sociais distintos, e através destes estudos é possível detectar em que medida é possível utilizar um instrumento originário de outro país, ou ainda, quais as alterações necessárias para o seu uso no novo contexto 
Para futuras investigações sugerem-se novas análises dos itens do Inventário que revelaram problemas, suprimindo aqueles que, por ventura, não sejam representativos na avaliação da auto-estima na realidade brasileira. O aperfeiçoamento deste instrumento ou a construção de um novo instrumento baseado neste estudo, que seja adequado à população brasileira, contribuirá para o progresso do estudo e avaliação da auto-estima, bem como para a prática profissional dos psicólogos.

\section{Referências}

Almeida, L. S., Prieto, G., Muñiz, J. \& Bartran, P. (1998). O uso dos teste em Portugal, Espanha e Países Ibero-Americanos. Psychological, 20, 2740.

Azevedo, M. M, Almeida, L. S, Pasquali, L. \& Veiga, H. M. S. (1996). Utilização dos testes psicológicos no Brasil: Dados de estudo prelimina em Brasilia. Em L. S. Almeida, S. Araújo, M. M. Gonçalves, C. Machado \& M. R. Simões (Orgs.), Avaliagão psicológica: Formas e contextos (Vol. IV pp. 213-219). Braga: Associação dos Psicólogos Portugueses.

Andrew, C. \& Tracy, N. (1996). First steps toward competence: promoting self-esteem and confidence in young children with disabilities. Em L. E. Powers, G. H. S. Singer \& J. Sowers (Orgs.), On the road to autonomy. promoting self-competence in cbildren and youth disabilities (pp. $373-387$ ).
Baltimore: Paul H. Brookes.

Bartram, D. (1999). International guidelines for the development of test-user performance standards. Version 4. 1: Second Consulation Draft (http: / c cwis. kub. nl/ $\sim f_{\text {sw }}$ 1/itc/itcfram4. btm). Recuperado em 27/01/1999.

Baumrind, D. (1967). Child care practices anteceding three patterns of preschool behavior. Genetic Psychology Monographs, 75, 43-88.

Bednar, R. L \& Peterson S. R. (1995). Self-esteem: Paradoxes and innovations in clinical theory and practice. Washington, American Psychological Association.

Bracken, B. A. (1996). Handbook of self-concept: Developmental, social, and clinical considerations. New York: Wiley.

Brown, L., \& Alexander, J. (1991). Self-Esteem Index. Austin, TX: Pro-Ed. Buss, A. H. (1995). Personality, social behavior and the self. Massachusets: Allyn

\& Bacon.
Coll, C., Palacios, J. \& Marchesi, A. (1995). Desenvolvimento psicológico e educacão: Psicologia evolutiva. Porto Alegre: Artes Médicas.

Coopersmith, S. (1967). The antecedents of self-esteem. San Francisco: Freeman Coopersmith, S. (1989). Coopersmith Self-Esteem Inventory. Palo Alto, CA Consulting Psychologists Press.

Cowan, R., Altmann, H. \& Pysh, F. (1978). A validity study of selected selfconcept instruments. Measurement and Evaluation in Guidance, 10, 211 221

Fitts, W. H. (1965). Tennessee Self-Concept Scale. Nashville. TN: Counselor Recordings and Tests.

Friedman, S., Gettys, J. \& Rogers, P. P. (1975). Project RE-DE: Increase in self-esteem as measured by the Coopersmith Inventory. Perceptual and Motor Skills, 40, 165-166.
Guzzo, R. S. L. (1999). T-CRS Adaptation Process for the Portuguese Version. Manuscrito não publicado. Departament of Social and Cognitive Psychology, University of Rochester, Rochester, USA

Guzzo, R. S. L, Gayotto, A. C. G, Messias, T. S. C. \& Silva, M. P. (1998). Auto-estima, autoconceito e desenvolvimento social: Estado da arte. Anais IV Encontro de Iniciasão Científica. Campinas: PUC-Campinas, SP.

Hambleton,R. K. (1994). Guidelines for adapting educational and psychological tests: A progress report. European Journal of Psychological Assessment, 10, 229-244.

Harter, S. (1993). Causes and consequences of low self-esteem in children and adolescents. Em L. E. Powers, G. H. S. Singer \& J. Sowers (Orgs.), and adolescents. Em L. E. Powers, G. H. S. Singer \& J. Sowers (Orgs.),
On the road to autonomy: Promoting self-competence in cbildren and youth disabilities On the road to autonomy: Promoting self-competence
(pp. 165-169). Baltimore: Paul H. Brookes.

Marsh, H. W. (1988). Self-Description Questionnaire, I. San Antonio, TX: The Psychological Corporation.

Marsh, H. W. (1990). Self-Description Questionnaire, II. San Antonio, TX: The Psychological Corporation.

Martins, S. R. S. (1997). Auto-conceito em crianscas repetentes e não repetentes: Dois procedimentos de avaliação. Dissertação de Mestrado não publicada. Curso de Pós-Graduação em Psicologia, Pontifícia Universidade Católica de Campinas, Campinas, São Paulo.

Mruck, C. (1998) . Auto-estima: Investigación, teoría y práatica . Bilbao: Desclée de Brouwer.

Nisbet, J. (1996) . The interrelationship of education and self-esteem. Em L. E. Powers, G. H. S. Singer \& J. Sowers (Orgs.), On the road to autonomy: Promoting self-competence in children and youth disabilities (pp. 165-169).
P. Bromoting self-competence in child

Piers, E. V. (1984). Piers-Harris Children's Self-Concept Scale: Revised manual. Los Angeles. CA: Western Psychological Services.

Poppovic, A., Esposito. Y. L. \& Cruz, L. M. C. (1973). Marginalização cultural: Uma metodologia para o seu estado. Cadernos de Pesquisa, 7, 5-6.

Powers, L. E., Singer, G. H. S \& Sowers, J. (1996). Self-competence and disability. Em L. E. Powers, G. H. S. Singer \& J. Sowers (Orgs.), On the road to autonomy: Promoting self-competence in cbildren and youth disabilities (pp. 3-24). Baltimore: Paul H. Brookes.

Rosenberg, M. (1979). Conceiving the self. New York: Basic Books.

Rosenberg, M. (1983). La auto-estima del adolescente y la sociedad. Buenos Aires: Paidós.

Sanches, G., Jiménez, F. Y. \& Merino, V. (1997). Auto-estima y autoconcepto en adolescentes: Una reflexión para la orientación educativa. Revista de Psicologia de la PUC, XV, 201-221.

Souza, Y. C. (1978). Auto-imagens de adolescentes de presidente prudente: Um estudo exploratório. Dissertação de Mestrado não publicada. Instituto de Psicologia da Universidade de São Paulo, SP.

Wells, E. L. \& Marwell, G. (1976). Self-esteem: Its conceptualization and measurement. California: Sage.

Wyllie, R. C. (1974). The self-concept: A review of methodological considerations and measuring instruments. Lincoln: University of Nebraska.

Sobre as autoras

Mônica Gobitta é Doutoranda e Mestre em Psicologia Escolar pela PUC Campinas. Integrante do

LAMP - Laboratório de Avaliação e Medidas em Psicologia. Bolsista CNPq.

Raquel Souza Lobo Guzzo é Professora Titular do Instituto de Psicologia da Pontifícia Universidade

Católica de Campinas. Doutora em Psicologia Escolar pela Universidade de São Paulo. Coordenadora

do LAMP - Laboratório de Avaliação e Medidas em Psicologia. Pesquisadora do CNPq. 